\title{
Memory of Occasional Events in Rats: Individual Episodic Memory Profiles, Flexibility, and Neural Substrate
}

\author{
Alexandra Veyrac, ${ }^{1,2,3}$ Marina Allerborn, ${ }^{3 *}$ Alexandra Gros, ${ }^{1,2 *}$ - Frederic Michon, ${ }^{3}$ Louise Raguet, ${ }^{3}$ Jana Kenney, ${ }^{3}$ \\ Florette Godinot, ${ }^{3}$ Marc Thevenet, ${ }^{3}$ Samuel Garcia, ${ }^{3}$ Belkacem Messaoudi, ${ }^{3}$ Serge Laroche,,${ }^{1,2} \dagger$ and $\odot$ Nadine Ravel $^{3} \dagger$ \\ ${ }^{1}$ Centre National de la Recherche Scientifique, Centre de Neurosciences Paris-Sud, ${ }^{2}$ Université Paris-Sud, Unité Mixte de Recherche 8195, F-91405 Orsay, \\ France, ${ }^{3}$ Unité Mixte de Recherche 5292, Centre National de la Recherche Scientifique, Institute national de la santé et de la recherche médicale U1028, \\ University Lyon 1, Lyon Neuroscience Research Center, F-69366 Lyon, France
}

In search for the mechanisms underlying complex forms of human memory, such as episodic recollection, a primary challenge is to develop adequate animal models amenable to neurobiological investigation. Here, we proposed a novel framework and paradigm that provides means to quantitatively evaluate the ability of rats to form and recollect a combined knowledge of what happened, where it happened, and when or in which context it happened (referred to as episodic-like memory) after a few specific episodes in situations as close as possible to a paradigm we recently developed to study episodic memory in humans. In this task, rats have to remember two odor-drink associations (what happened) encountered in distinct locations (where it happened) within two different multisensory enriched environments (in which context/occasion it happened), each characterized by a particular combination of odors and places. By analyzing licking behavior on each drinking port, we characterized quantitatively individual recollection profiles and showed that rats are able to incidentally form and recollect an accurate, long-term integrated episodic-like memory that can last $\geq 24 \mathrm{~d}$ after limited exposure to the episodes. Placing rats in a contextually challenging recollection situation at recall reveals the ability for flexible use of episodic memory as described in humans. We further report that reversible inactivation of the dorsal hippocampus during recall disrupts the animal's capacity to recollect the complete episodic memory. Cellular imaging of c-Fos and Zif268 brain activation reveals that episodic memory recollection recruits a specific, distributed network of hippocampal-prefrontal cortex structures that correlates with the accuracy of the integrated recollection performance.

Key words: episodic-like memory; hippocampus; olfactory memory; prefrontal cortex; recollection; rodent

\section{Introduction}

In humans, episodic memory is characterized as the conscious recollection of unique personal past experiences, often occurring singly during daily life, including what happened, where the event happened, and when it happened (Tulving, 1972). Later refined

Received Sept. 16, 2014; revised March 2, 2015; accepted April 6, 2015.

Author contributions: A.V., B.M., S.L., and N.R. designed research; A.V., M.A., A.G., F.M., L.R., J.K., F.G., and N.R. performed research; A.V., M.A., A.G., F.M., L.R., J.K., F.G., M.T., S.G., B.M., and N.R. analyzed data; A.V., S.L., and N.R. wrote the paper.

This work was supported by Centre National de la Recherche Scientifique, University Lyon 1, University Paris-Sud 11, and by grants from the Agence Nationale de la Recherche (ANR-2010-BLAN-1413-01) to N.R. and S.L. (postdoctoral fellowship to A.V. and J.K.), and by the Labatoire d'Excellence Cortex (ANR-11-LABX-0042) within the program Investissements d'Avenir (ANR-11-IDEX-0007; postdoctoral fellowship to A.V.). M.A. and A.G. were supported by doctoral fellowships from the French Ministry of Research. We are grateful to F. Esclassan, B. Poucet, V. Hok, and E. Save for helpful discussions; to D. Meunier for his help in correlation analyses; and to 0. Ben-hellal, F. Lhericel, C. Dubois, and P. Veyrac for animal care.

The authors declare no competing financial interests.

*M.A. and A.G. contributed equally to this work.

†S.L. and N.R. contributed equally to this work.

Correspondence should be addressed to either of the following: Dr. Nadine Ravel, Centre de Recherche en Neurosciences de Lyon, CNRS UMR 5292-INSERM U1028-Université Lyon1, Equipe Olfaction du codage à la mémoire, 69366 Lyon cedex, France. E-mail: nadine.ravel@cnrs.fr; or Dr. Serge Laroche, Centre de Neurosciences Paris-Sud (CNPS), CNRS UMR8195, UniversitéParis Sud, Bat. 446, 91405 Orsay cedex, France. E-mail: serge.laroche@u-psud.fr.

A. Veyrac's present address: Centre de Recherche en Neurosciences de Lyon, CNRS UMR 5292-INSERM U1028Université Lyon1, Equipe Olfaction du codage à la mémoire, 69366 Lyon cedex, France.

DOI:10.1523/JNEUROSCI.3941-14.2015

Copyright $\odot 2015$ the authors $\quad 0270-6474 / 15 / 337575-12 \$ 15.00 / 0$ by introducing autonoetic awareness (remembering personal experiences with the awareness of reliving the events; Tulving, 2002), the initial concept stimulated the development of new tasks to probe episodic memory in animals, an essential condition to advance our understanding of the neurobiological mechanisms underlying episodic memory and associated disorders.

The first evidence for "episodic-like memory" in animals was provided by Clayton and Dickinson (1998) reporting that scrub jays were naturally expressing this type of memory during foodcaching behavior, since they were able to remember where and when they cached a particular type of food (what). This pioneer study inspired the design of behavioral tasks to probe episodiclike memory in rodents (Zhou and Crystal, 2011; Fellini and Morellini, 2013). These studies confirmed that rodents can form an integrated memory for what-where-when in certain experimental situations; however, the temporal component of memory and the efficient way to assess it in animal models still remain an open debate (Suddendorf and Corballis, 2007). In most studies, however, the when component is addressed in a way that is indissociable from where or what information, and therefore might more reflect differences in memory strength for what was seen where, than strictly the temporal component of episodic memory (Easton and Eacott, 2008, 2010; Eacott and Easton, 2010; Templer and Hampton, 2013). One alternative is to consider that the 
when component of episodic memory is represented more by the capacity to order events than by precisely dating them (Ergorul and Eichenbaum, 2004). These experiments, however, require that animals be extensively trained before they can extract a rule and become able to order events. Moreover, to perform this time reconstruction, humans most often use event-related information or contextual elements: "The last time I went to Paris, I visited my friend in her new house and we talked in the garden until midnight, so it must have been in summer." Thus, as proposed by others (Eacott et al., 2005; Easton and Eacott, 2008), replacing "when" with "in which occasion/context" represents an efficient way to test episodic memory, as we recently showed in a novel episodic memory paradigm in humans (Saive et al., 2013, 2014).

Based on this, we designed a paradigm allowing precise evaluation of the ability of rodents to form long-term integrated episodic memory in a manner as close as possible to humans in terms of informational content, limited occasional encounters of episodes, and the capacity for flexible use of this memory in a challenging environment. With this original protocol, we found that rats can form a long-term episodic-like memory and remember that, during their daily life, in a given multisensory-enriched environment (in which context), a particular odor (what) at a specific location (where) signals something pleasant or unpleasant to drink. We provided the first characterization and categorization of individual profiles of episodic memory recollection in rats, showing how strikingly individual successes/errors compare with human episodic memory recollection, and show that rats can form a long-term episodic memory lasting $\geq 24 \mathrm{~d}$. We confirmed that hippocampal inactivation impairs episodic memory recollection and found by mapping c-Fos and Zif268 immediate early gene activation that episodic-like memory recruits a large, distributed hippocampal-prefrontal cortex (PFC) network that correlates with the accuracy of long-term episodic memory recollection.

\section{Materials and Methods \\ Animals}

Experiments were performed in accordance with European Directive 86/609/EEC regarding the care and use of laboratory animals. Adult male Long-Evans rats (Charles River Laboratories; 7 weeks old, 300-350 g at the start of the experiment; $n=65$ for the whole study) were housed in a temperature-controlled and humidity-controlled colony room in groups of 2-4 during the shaping procedure. They were exposed to a $12 \mathrm{hlight} /$ dark cycle (light onset, 6:00 A.M.) and experiments were conducted during the light period (between 9:00 A.M. and 5:00 P.M.). Food was available ad libitum but access to water was progressively restricted (20-40 $\mathrm{min}$ in the experimental cage and 40 additional minutes in home cages at 6:00 P.M.). Rats were weighed weekly to control their adaptation to hydric restriction.

\section{Apparatus: the Episodicage}

The experimental arena (Fig. 1A), the Episodicage (designed by Belkacem Messaoudi), consisted of a rectangular polyvinyl chloride box $(60 \times$ $35 \times 40 \mathrm{~cm}$ ) with devices allowing delivery of various odors and drinking solutions. Two walls of the arena were each equipped with two odor ports (Fig. 1B). Deodorized air constantly flowed through the odor ports. Detection of a rat nose poke by a capacitance system triggered odor delivery through a custom-designed olfactometer. A vacuum system ensured that the odor remained confined to the port. A hole in the wall, $1 \mathrm{~cm}$ below each odor port, allowed introduction or withdrawal of a drinking pipette. Each lick to a pipette was detected by another capacitance system to trigger a pump that delivered a calibrated volume of solution (Fig. 1C). The whole device configuration ensured that the odor stimulation was delivered through the port during the entire liquid consumption, allow-

\section{A}

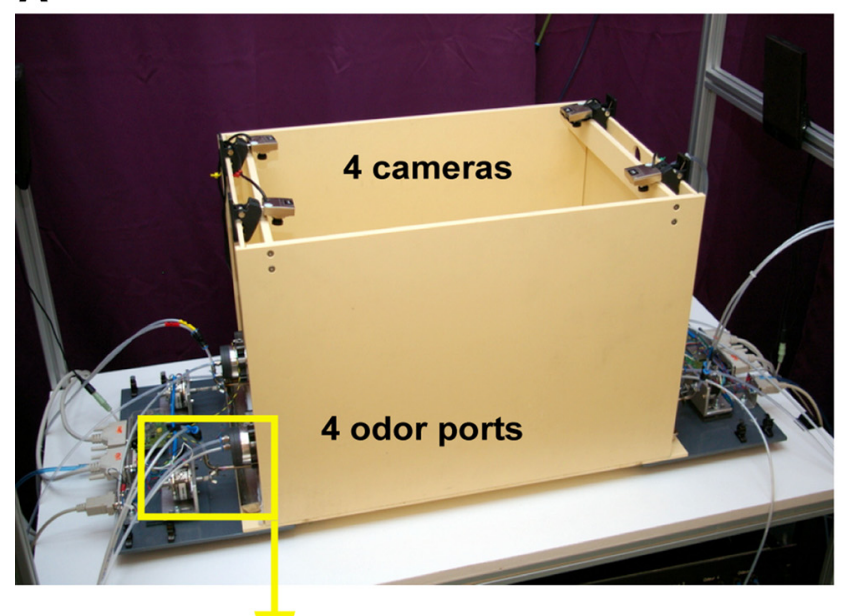

B

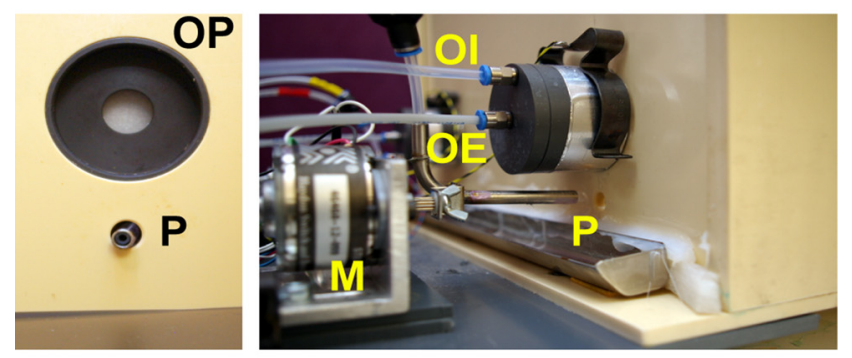

C

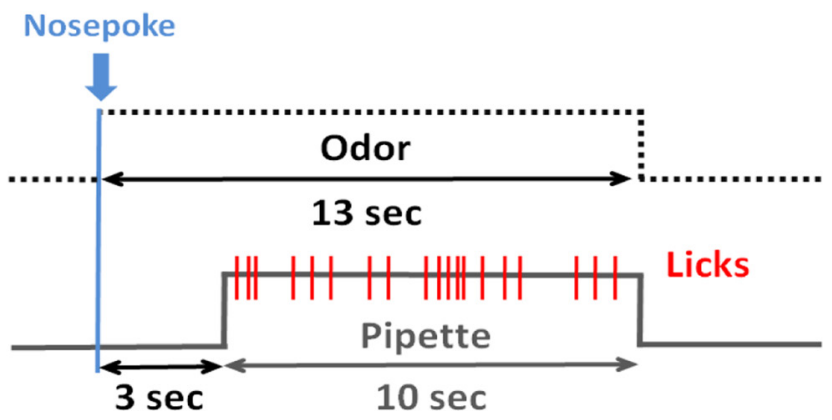

Figure 1. The Episodicage: an experimental device to study episodic-like memory in rats. $\boldsymbol{A}$, $\boldsymbol{B}$, The Episodicage is a rectangular chamber with four odor ports (OP), each associated with a drinking pipette $(P)$. Detection of rat's nose poke triggers odor delivery $(0 l$, odor injection; $0 \mathrm{E}$, odor extraction) and introduction/withdrawal of the pipette by a motor (M). Rat behavior is tracked by four cameras above the odor ports and one central camera above the arena. The appearance of Episodicage could be modified by placing floors with different tactile characteristics, introducing various objects, using a video projector to project visual patterns on different versions of the floor, and diffusing environmental sounds via loudspeakers. C, A nose poke initiates the trial and the onset of the odor. After a delay, the pipette protrudes into the cage for a fixed amount of time. During this period, each lick to the pipette is detected and triggers a pump for delivery of drinking solutions during odor stimulation. The offset of the odor corresponds to the pipette withdrawal. After a given intertrial interval, the rat can activate the system again by a nose poke and a new trial is initiated.

ing perfect coupling between the odor and the consumed solution. Each odor port was controlled by a computer that fixed various parameters: the quality, intensity, and duration of the olfactory stimulation, as well as the delay between odor delivery onset and availability of the pipette. This system recorded the number of licks detected on any pipette for a given visit and reconstructed their temporal distribution (Fig. 1C). The standard arena floor could be replaced to introduce materials differing from their tactile characteristics. Various objects could also be placed in the 


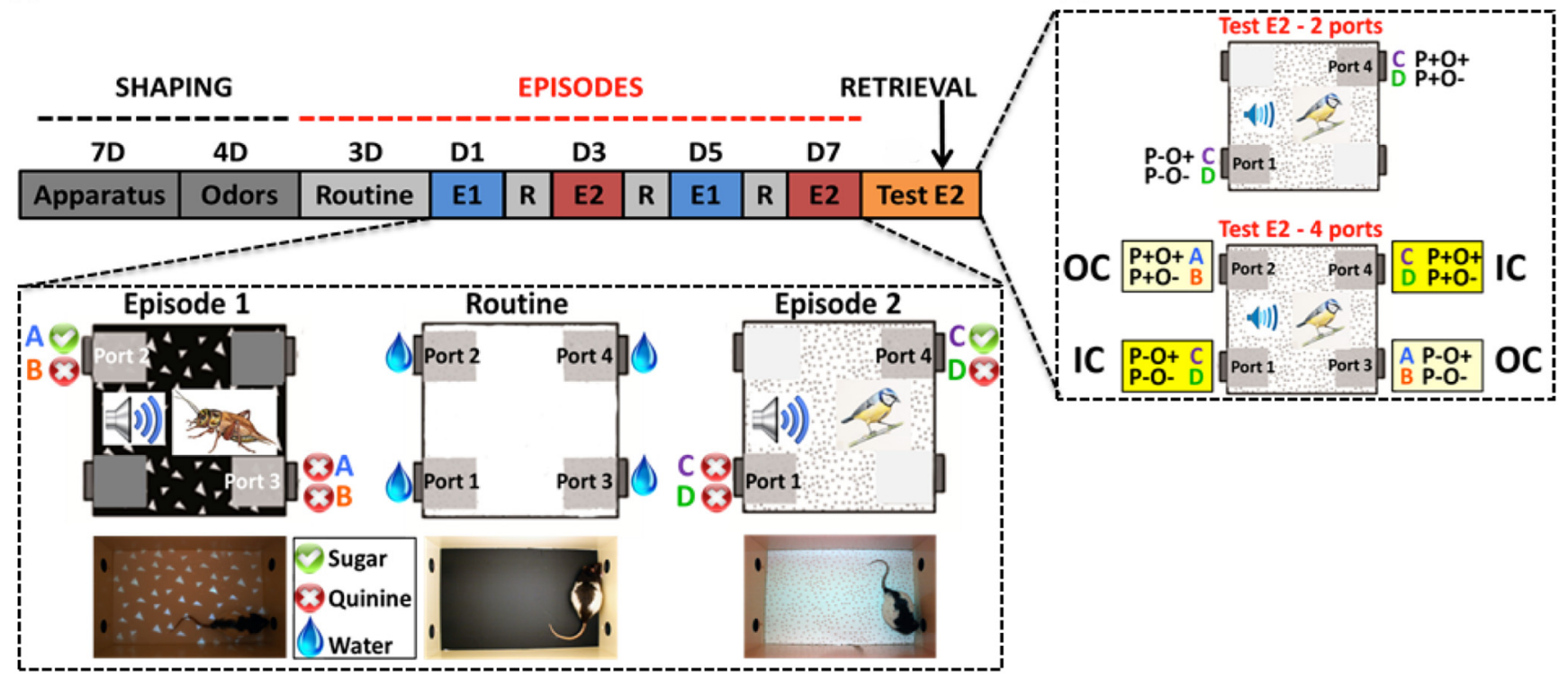

Figure 2. Time line of the episodic-like memory paradigm. $A$, First, 11 daily sessions of shaping were used to train the animal to the introduction of the pipette by making a nose poke into any of the four odor ports and drink after a delay (apparatus, $7 \mathrm{~d}$ ) and to receive odor stimulation associated with sugar or quinine solution from the pipette (odors, $4 \mathrm{~d}$ ). All trials were systematically initiated by the rats during a $20 \mathrm{~min} / \mathrm{d}$ session. Rats then underwent three daily routine sessions (Routine, lower box, middle configuration) with no odor, no enriched context, and only water available from the four ports. Episode exposures consisted of two distinct episodes [lower box: left, Episode 1 (E1) configuration; right, Episode 2 (E2) configuration] with a $1 \mathrm{~d}$ routine (R) session in between. Episodes were presented once or twice (as illustrated here) in independent groups of rats. They were each characterized by a unique combination of odor-place-context associations rewarded with sugar solution (green labels; odor A at port 2 for E1; odor ( at port 4 for E2) while the three other incorrect odor-place associations were associated with quinine (red crosses). During this episodic session, in a given context ("in which context"), rats encoded at which port location ("where"), one of the odors ("what") was associated with sugar solution. $\boldsymbol{B}$, During the retrieval test $24 \mathrm{~h}$ or $24 \mathrm{~d}$ after the last episode session, rats were placed again in the $\mathrm{E} 2$ context to evaluate what type of information they were able to recollect $(\mathrm{P}+0+:$ correct place, correct odor; $\mathrm{P}+0-$ : correct place, incorrect odor; $\mathrm{P}-0+$ : incorrect place, correct odor; $\mathrm{P}-0-$ : incorrect place, incorrect odor). The two-port test completely matched the episode, except that only water was delivered whatever place- odor configuration was experienced. The challenging four-port test was a more complex situation since it took place in the enriched context E2, but with four accessible ports [2 previously associated with context E2 (IC) and 2 with context E1 (OC)], each port associated with the pair of odors corresponding to its respective episode. For each port visit, the number of licks was expressed for each rat as a licks index and was used for analyzing encoding and recollection performance.

arena. The appearance of the arena during the episodes was also modified by using a video projector that projected visual patterns on the different versions of the floor (Fig. 2A). Two loudspeakers symmetrically placed above the experimental arena were used to provide different sound environments during the episodes. The rats' behavior was monitored by five cameras: one placed centrally above the arena and four others above the four different odor ports (Fig. 1A). The video signals were acquired throughout each session. A video tracking software (Volcan, designed by Marc Thevenet) was used to detect on-line the position of the animal and trigger the camera corresponding to the activated port.

\section{Behavioral procedure}

Shaping

Shaping consisted of two different steps achieved in an average of 11 daily sessions lasting $20 \mathrm{~min}$ at maximum (Fig. 2). The objective of step 1 (apparatus shaping; $7 \mathrm{~d}$ ) was to initiate the animal to the introduction of the pipette by making a nose poke deep into any of the four odor ports and to drink water. On the first day, water-deprived animals were allowed to explore the arena. Pipettes from all ports were already introduced into the arena and delivered water upon licking. During the next $3 \mathrm{~d}$, animals learned to trigger the pipette entry into the arena via a nose poke in the odor port. The pipette was delivered immediately after a nose poke and remained available for drinking for $20 \mathrm{~s}$ ( progressively reduced to $10 \mathrm{~s}$ ), after which it was withdrawn and the rat could then initiate the next trial after a refractory period of $16 \mathrm{~s}$. The following $3 \mathrm{~d}$, a $2 \mathrm{~s}$ delay was introduced between the nose poke and pipette entry.

In step 2 (odor shaping; $4 \mathrm{~d}$ ), rats were habituated to receive odor stimulation associated with sugar or quinine solution from the pipette. Two different odors, geraniol and eugenol (Sigma-Aldrich) were released upon nose poke for $10 \mathrm{~s}$ from each of the four odor ports and were associated with quinine $(0.06 \%)$ or sugar $(6 \%)$. Each daily session was identical and the odor of geraniol or eugenol was introduced at the four odor ports in a pseudorandom manner so that each animal was exposed to the same amount of both odors. The delay between a nose poke and pipette delivery was then increased ( $3 \mathrm{~s}$ ) to maintain the attention to the odor after the nose poke. This step was terminated when $\geq 15$ trials in 20 min were performed. The aim was to give the animals a hint that a given odor might be an indicator for finding a sweet or avoiding a bitter drinking solution, which otherwise could not be predicted.

\section{Episodic-like memory task}

Routine sessions. During the 3 following days, before episode exposures, water-deprived rats spent $15 \mathrm{~min} / \mathrm{d}$ (or $\geq 12$ trials) in the Episodicage. The appearance of the arena was exactly the same as during the shaping period (Fig. 2A). Neither visual nor auditory enrichment of the context was used during routines and the floor was the standard one (black and smooth). The four ports were accessible to nose poke. However, no odors were released and pipettes delivered water for $10 \mathrm{~s}$. The aim of routine sessions was to enhance the salience of the following episodes and to control for an evenly distributed port-visiting behavior of the rats before episode exposures.

Episode exposures. Two different episodes, Episode 1 (E1) and Episode 2 (E2), were used and presented to the rats separated by $1 \mathrm{~d}$ of routine session. Episode sessions were presented once or repeated once in independent groups of rats, depending on the experiment (see below). Each episode was characterized by enriched contextual information consisting of distinct flooring, specific visual patterns projected on the floor, and distinct natural sounds diffused during each episode session (Fig. 2A). Visually, we favored highly contrasted black and white patterns. The environmental sounds were selected for their broad sensory information content. The capacity of rats to hear and discriminate these sounds was validated by analyzing dominant frequency and amplitude modulation on spectrograms. In each episode, a nonoverlapping configuration of only two ports was accessible, each of them releasing two different odors 
unique to each episode (i.e., odors $\mathrm{A}$ and $\mathrm{B}$ in $\mathrm{E} 1$ at ports 2 and 3 and odors $\mathrm{C}$ and $\mathrm{D}$ in $\mathrm{E} 2$ at ports 1 and 4; Fig. 2A). For a given episode, one of the two ports only delivered sugar or quinine depending on the odor triggered in the port, while the other port delivered quinine solution regardless of the odor that was triggered following a nose poke. Thus, each episode was characterized by one unique combination of odorplace-context association positively rewarded by sugar solution $(\mathrm{P}+\mathrm{O}+$ : correct place, correct odor, i.e., odor $\mathrm{A}$ at port 2 in context used for E1 and odor $\mathrm{C}$ at port 4 in context used for E2; Fig. $2 A$, green label), while the three other odor-place associations were associated with quinine $(\mathrm{P}+\mathrm{O}-$ : correct place, incorrect odor; $\mathrm{P}-\mathrm{O}+$ : incorrect place, correct odor; $\mathrm{P}-\mathrm{O}-$ : incorrect place, incorrect odor; Fig. $2 \mathrm{~A}$, red cross). The odors used, carvone (odor A) and isoamyl acetate (odor B) for E1 and anethol (odor C) and citral (odor D) for E2, were chosen according to data obtained in previous experiments. Each pair was easy to discriminate and both pairs of odors were spontaneously equally explored at the selected concentration. Each episode session included 24 trials combined in a pseudorandom sequence including six repetitions (three in the first 12 and three in the following 12 spontaneous trials) of four different configurations (i.e., $\mathrm{P}+\mathrm{O}+$ vs $\mathrm{P}-\mathrm{O}+; \mathrm{P}+\mathrm{O}+$ vs $\mathrm{P}-\mathrm{O}-; \mathrm{P}+\mathrm{O}-$ vs $\mathrm{P}-\mathrm{O}-; \mathrm{P}+\mathrm{O}-$ vs $\mathrm{P}-\mathrm{O}+)$. For each session, the time assigned to the exploration during the episode was $\geq 20 \mathrm{~min}$ and limited to $40 \mathrm{~min}$, and the minimum number of trials was 12 . During this session, in a given enriched context ("in which context" information), rats encoded at which port ("where" information) which of the odors ("what" information) was associated with sugar solution.

Retrieval test. A test session was performed $24 \mathrm{~h}$ after the last episode exposure to evaluate what type of information the rats were able to recollect (Fig. 2B). During the test (12 trials), rats were replaced in the E2 context. Two versions of the test that differed in their levels of difficulty were carried out. The two-port test (Fig. 2B, top) was the easiest because the test situation matched completely the episode in terms of context, available ports, and odors, except that only water was delivered regardless of the place and odor. The four-port test (Fig. 2B, bottom) was more complex since it took place in the E2 context, but for the first time the four ports were accessible [those used during E2: in context (IC); those corresponding to E1: out of context (OC)] and the two odors from both previous episodes were simultaneously present and available from their respective ports. Again, at test, only water was delivered upon nose pokes. To test memory at a much longer training-to-retention interval, a further group of rats was trained and performance in the two-port retrieval test was examined $24 \mathrm{~d}$ after the last exposure to $\mathrm{E} 2$.

\section{Behavioral analysis}

We quantified the number of visits on each port for each experimental configuration, including routine sessions, and expressed it as a ratio of the total number of trials in a given session. During exposure to the episodes, this ratio was used to estimate how often each odor-place combination was experienced. For each port visit, the number of licks was normalized for each rat to the total number of licks in a given session to normalize individual licking behavior differences. This value was referred to as the licks index and was used for the analysis of performance. During routines, this variable was used to verify that intake was similar from any of the four pipettes. During exposure to the episodes, it served to evaluate behavioral discrimination between $\mathrm{P}+\mathrm{O}+$ and the other three odor-place associations $(\mathrm{P}+\mathrm{O}-; \mathrm{P}-\mathrm{O}+; \mathrm{P}-\mathrm{O}-)$. During the retrieval test, the licks index allowed us to evaluate precisely what the rats expected in a given configuration and therefore what type of information encoded during the episode they were able to recall. In the two-port test, we compared the licks index for the four odor-place configurations with expected licks index values for archetype profiles of rats (Table 1 ). If the licks index for $\mathrm{P}+\mathrm{O}+$ was higher than that of the three other configurations (close to an archetype rat with 1.0 for $\mathrm{P}+\mathrm{O}+, 0$ for $\mathrm{P}+\mathrm{O}-, 0$ for $\mathrm{P}-\mathrm{O}+, 0$ for $\mathrm{P}-\mathrm{O}-$ ), we concluded that the animal was able to recall a combination of "what" and "where" information (WW profile) in a given context (close to the archetype 0.5 for $\mathrm{P}+\mathrm{O}+, 0.5$ for $\mathrm{P}+\mathrm{O}-, 0$ for $\mathrm{P}-\mathrm{O}+, 0$ for $\mathrm{P}-\mathrm{O}-$ ). If this index was different for $\mathrm{P}+$ configurations compared with $\mathrm{P}-$ but without any difference between $\mathrm{P}+\mathrm{O}+$ and $\mathrm{P}+\mathrm{O}-$, we concluded that only the "where" information was recalled
Table 1. Examples of licks index values and corresponding individual recall profiles in the two-port test

\begin{tabular}{|c|c|c|c|c|c|c|}
\hline \multirow[b]{2}{*}{ Presentation } & \multirow{2}{*}{$\begin{array}{l}\text { Individual } \\
\text { profiles }\end{array}$} & \multirow{2}{*}{$\begin{array}{l}\text { Percentage } \\
\text { rats }\end{array}$} & \multicolumn{4}{|c|}{ Licks index per configuration } \\
\hline & & & $\mathrm{P}+0+$ & $P+0-$ & $\mathrm{P}-0+$ & $P-0-$ \\
\hline One episode & What-where ${ }^{a}$ & $29 \%$ & 0.92 & 0.07 & 0 & 0.01 \\
\hline \multirow{3}{*}{ presentation } & Where $^{b}$ & $43 \%$ & 0.58 & 0.42 & 0 & 0 \\
\hline & What $^{c}$ & $14 \%$ & 0.48 & 0.19 & 0.26 & 0.07 \\
\hline & Indeterminate $^{d}$ & $14 \%$ & 0.04 & 0.41 & 0.55 & 0 \\
\hline $\begin{array}{l}\text { Two episode } \\
\text { presentations }\end{array}$ & What-where $^{a}$ & $100 \%$ & 0.98 & 0.01 & 0.01 & 0 \\
\hline
\end{tabular}

${ }^{a}$ Profile with more licks in $\mathrm{P}+0+$ of the tested context.

${ }^{b}$ Profile with similar licks in $\mathrm{P}+0+$ and $\mathrm{P}+0-$.

'Profile with similar licks in $\mathrm{P}+0+$ and $\mathrm{P}-0+$

${ }^{d}$ Atypical profile.

(Where profile). Symmetrically, a significantly higher licks index for $\mathrm{O}+$ compared with $\mathrm{O}-(0.5$ for $\mathrm{P}+\mathrm{O}+, 0$ for $\mathrm{P}+\mathrm{O}-, 0.5$ for $\mathrm{P}-\mathrm{O}+, 0$ for $\mathrm{P}-\mathrm{O}-)$ regardless of the port where it has been delivered signified retrieval of "what" information (What profile). Similar licks index for each configuration indicated that no information was retrieved (indeterminate behavior; 0.25 for $\mathrm{P}+\mathrm{O}+, 0.25$ for $\mathrm{P}+\mathrm{O}-, 0.25$ for $\mathrm{P}-\mathrm{O}+, 0.25$ for $\mathrm{P}-\mathrm{O}-$ ). In the four-port test (Table 2), the number of possible configurations was increased since in a given context it was possible for the rat to activate the ports corresponding to the tested context (IC) or those associated to the other context, used as distractors (OC). In that case, we first determined whether the licks index was superior for the ports of the tested context or for the distractor context. This meant that the animal was able to retrieve the association between the ports and the context and animals were classified as IC, while those for which the licks index was superior for the distractor context were classified as OC. Then, the same analysis as above for the two-port test was used to categorize the recall profile of each animal. As an example, an animal licking only on the $\mathrm{P}+\mathrm{O}+$ associated to the tested context was considered as retrieving the overall information presented during exposure to the episode sessions and was classified as what-where-IC. With this classification, we determined the proportion of each profile in the group.

Licks index data were averaged across animals within each experimental group and are presented as means \pm SEM. Statistical comparisons between configurations were conducted by the nonparametric Friedman test followed by a Wilcoxon test. Data comparisons from independent groups were assessed by nonparametric Mann-Whitney and KruskallWallis tests. Significance level was set at $p<0.05$.

\section{Surgery and hippocampal inactivation procedures}

A few days after shaping, rats $(n=17)$ were anesthetized (equithesin: mixture of $29.6 \mathrm{mg} / \mathrm{kg}$ pentobarbital and $141.3 \mathrm{mg} / \mathrm{kg}$ chloral hydrate for induction, supplemented by $8.9 \mathrm{mg} / \mathrm{kg}$ and $42.4 \mathrm{mg} / \mathrm{kg}$, respectively) and a specific procedure was applied to ensure proper analgesia (local anesthetic agents combined to nonsteroidal anti-inflammatory drugs). Using standard stereotaxic procedures, guide microtubes (diameter, 23 gauge; length, $12 \mathrm{~mm}$; Phymep) were bilaterally implanted $500 \mu \mathrm{m}$ above the CA1 pyramidal layer of the dorsal hippocampus $(3.6 \mathrm{~mm}$ posterior to bregma; $2.0 \mathrm{~mm}$ lateral; $2.0 \mathrm{~mm}$ ventral to dura). Guide tubes were fixed to the skull with dental cement and two anchoring screws. After surgery a removable stainless steel stylet was inserted into the tube, protruding from the tube by $1 \mathrm{~mm}$ to prevent obstruction and infection. Rats were placed individually in home cages for a 2 week recovery period with ad libitum food and water, during which the stylets were removed, disinfected, and replaced every $2 \mathrm{~d}$ to habituate the animals to this manipulation. Rats were then randomly assigned to three experimental groups. A first group $(n=7)$ received bilateral injections of the $\mathrm{GABA}_{\mathrm{A}}$ receptor agonist muscimol, dissolved in artificial CSF (aCSF; $0.5 \mu \mathrm{g} / \mathrm{L}$ ). A second group received aCSF only $(n=5)$ and the third group was implanted but not injected $(n=5)$. For microinjection, the stylets were removed and injection needles (diameter, 30 gauge) were inserted into the guide tubes with the needle tip protruding $\sim 1 \mathrm{~mm}$ beyond the guide extremity. The needle was connected to a $10 \mu \mathrm{l}$ Hamilton syringe and $0.5 \mu \mathrm{l}$ per site of 
Table 2. Examples of licks index values and corresponding individual recall profiles in the four-port test

\begin{tabular}{|c|c|c|c|c|c|c|c|c|c|}
\hline \multirow[b]{3}{*}{ Individual profiles } & \multirow[b]{3}{*}{ Percentage rats } & \multicolumn{8}{|c|}{ Licks index per configuration } \\
\hline & & \multicolumn{4}{|l|}{ IC } & \multicolumn{4}{|l|}{$O C$} \\
\hline & & $\mathrm{P}+0+$ & $P+0-$ & $\mathrm{P}-0+$ & $\mathrm{P}-0-$ & $\mathrm{P}+0+$ & $\mathrm{P}+0-$ & $\mathrm{P}-0+$ & $\mathrm{P}-0-$ \\
\hline What-where-IC ${ }^{a}$ & $50 \%$ & 0.70 & 0.07 & 0 & 0 & 0.23 & 0 & 0 & 0 \\
\hline Where-IC $C^{b}$ & $20 \%$ & 0.41 & 0.40 & 0 & 0 & 0.19 & 0 & 0 & 0 \\
\hline What-IC & $10 \%$ & 0.36 & 0.10 & 0.33 & 0 & 0.21 & 0 & 0 & 0 \\
\hline What-where-0 ${ }^{d}$ & $10 \%$ & 0 & 0.05 & 0 & 0 & 0.95 & 0 & 0 & 0 \\
\hline Where- $0 C^{e}$ & $10 \%$ & 0.17 & 0 & 0 & 0 & 0.33 & 0.32 & 0.18 & 0 \\
\hline
\end{tabular}

${ }^{a}$ Profile with more licks in $\mathrm{P}+0+\mathrm{IC}$.

${ }^{b}$ Profile with similar licks in $\mathrm{P}+0+$ and $\mathrm{P}+0-\mathrm{IC}$.

cProfile with similar licks in $\mathrm{P}+0+$ and $\mathrm{P}-0+\mathrm{IC}$.

${ }^{d}$ Profile with more licks in $\mathrm{P}+0+O C$.

${ }^{e}$ Profile with similar licks in $\mathrm{P}+0+$ and $\mathrm{P}+\mathrm{O}-\mathrm{OC}$.

muscimol or aCSF were infused bilaterally in $30 \mathrm{~s}$. The needles were left in place for $2 \mathrm{~min}$ after the injection. Animals underwent the behavioral test 40 min later. Rats of the noninjected group were constrained for 4 min, similarly to the other rats during the injections.

At the end of the experiment, rats were injected with a lethal dose of pentobarbital and brains were removed and postfixed in $10 \%$ formaldehyde solution for 2 weeks. They were then cryoprotected by immersion in a $25 \%$ sucrose solution. Guide tube placement was determined by serial coronal sections ( $40 \mu \mathrm{m}$ thick) cut with a freezing microtome $\left(-20^{\circ} \mathrm{C}\right)$ and stained with cresyl violet. Behavioral data were analyzed only from rats with correct cannula placement.

\section{Immunochemistry and cellular imaging}

To evaluate c-Fos and Zif268 expression in brain areas specifically related to episodic-like memory, control rats were submitted to the same experimental protocol as episodic rats (Fig. 2), including shaping phases, except that they were exposed to routine sessions instead of episode sessions both during encoding and retrieval. Routine and episodic rats were deeply anesthetized with pentobarbital $(200 \mathrm{mg} / \mathrm{kg}$, i.p.) and perfused transcardially with a solution containing $4 \%$ paraformaldehyde in $0.1 \mathrm{M}$ phosphate buffer, $90 \mathrm{~min}$ after completion of the two-port recall test or a routine session (Fig. 2). While basal levels of Zif268 and c-Fos are sensitive to environmental events and novelty, behavioral procedures for episodic and routine rats on the test day matched as closely as possible those used during encoding and any environmental changes between testing and killing of animals was avoided. After perfusion, brains were removed and fixed overnight in the same perfusion solution at $4^{\circ} \mathrm{C}$, immersed for $6 \mathrm{~d}$ in phosphate buffer containing 30\% sucrose, and frozen in chilled 2-methylbutane $\left(-30^{\circ} \mathrm{C}\right)$. Coronal sections ( $14 \mu \mathrm{m}$ thick) were cut on a cryostat (Microm) from the olfactory bulb to the posterior part of the hippocampus (bregma 7.56 to $-6.48 \mathrm{~mm}$ ).

Brain sections were preincubated in Target Retrieval Solution (Dako) for $20 \mathrm{~min}$ at $95^{\circ} \mathrm{C}$. After cooling for $20 \mathrm{~min}$, sections were treated with $0.5 \%$ Triton in PBS for $30 \mathrm{~min}$ and then endogenous peroxidases were blocked with a solution of $3 \% \mathrm{H}_{2} \mathrm{O}_{2}$ in $0.1 \mathrm{M}$ PBS. Sections were then incubated for $90 \mathrm{~min}$ in 7.5\% normal serum (Jackson Immunoresearch), 2\% BSA (Sigma-Aldrich), and $0.1 \%$ Triton X-100 to block nonspecific binding, and incubated overnight at $25^{\circ} \mathrm{C}$ in Zif268-specific (1:1000) or c-Fos-specific (1:3000) primary rabbit polyclonal antibodies (Santa Cruz Biotechnology). Sections were then incubated in a goat biotinylated antirabbit secondary antibody (1:200; Vector Laboratories) for $2 \mathrm{~h}$ at room temperature and processed with avidin-biotin-peroxydase complex (1: 200; ABC Elite Kit, Vector Laboratories) for $30 \mathrm{~min}$. Finally, peroxidase detection was conducted with 3,3-diaminobenzidine-tetrahydrochloride $\left(0.06 \%\right.$; Sigma-Aldrich) as chromogen with nickel $\left(0.03 \% \mathrm{NiCl}_{2}\right)$ and $\mathrm{H}_{2} \mathrm{O}_{2}(0.06 \%)$ to obtain black immunolabeling. Sections were dehydrated in graded ethanols and coverslipped in Eukitt (Sigma-Aldrich).

Quantitative imaging analyses of c-Fos and Zif268 were conducted with a mapping software (Mercator Pro, Explora Nova) coupled to an optical microscope (Olympus) as previously described (Lesburguères et al., 2011; Veyrac et al., 2013). Cell counts were performed at $20 \times$ mag- nification and conducted by an experimenter blind to the experimental condition. Brain structures were anatomically defined according to the Paxinos and Watson atlas (see Fig. 6A). The numbers of c-Fos-positive and Zif268-positive nuclei were quantified in the following areas: medial, ventral, lateral, and dorsolateral parts of the orbital cortex (MO, VO, LO, DLO, respectively); prelimbic, infralimbic, anterior piriform, posterior piriform, anterior cingulate (aCC), medial cingulate (mCC), perirhinal, and lateral entorhinal cortices; and subfield CA1, subfield CA3, and dentate gyrus (DG) of the dorsal (dCA1, dCA3, dDG) and ventral hippocampus (vCA1, vCA3, vDG). Counting frames were placed in standardized manner based on predefined anatomic landmarks on sections (e.g., shape of cortex or of adjacent corpus callosum). c-Fos-positive and Zif268-positive neurons were counted in the entire quantification field and cell densities (number of c-Fos-positive and Zif268-positive nuclei per $\mu \mathrm{m}^{2}$ ) for each brain region were averaged for each experimental group.

Results were expressed as means \pm SEM. Since equality of variance $(F$ test) and normality of distribution (Shapiro-Wilk) were validated for all data, between-group parametric comparisons were performed by bilateral $t$ test. Only effects with a $p$ value $<0.05$ were considered as significant. Parametric Pearson's $r$ correlation coefficients were used to detect correlations between the density of c-Fos-positive or Zif268-positive nuclei and the licks index for $\mathrm{P}+\mathrm{O}+$ during the recall test. Pearson's $r$ correlation matrices were used to reveal cross-correlations between densities of c-Fos-positive and Zif268-positive nuclei in different brain areas.

\section{Results}

\section{Rats can form a long-term episodic-like memory of briefly encountered events}

The principle of the task was to briefly confront rats to two contextually distinct episodes in an otherwise familiar arena, named Episodicage (Figs. 1, 2). During each episode, defined by a specific multisensory contextual appearance of the arena, a nose poke in one defined port can deliver a specific odor upon which licking provides a positive reinforcement (Fig. 2). On this same port, a nose poke can trigger another odor, but one associated with a negative reinforcement (quinine) upon licking, which the rat should avoid doing. In the same context, the same two odors can be delivered on another port, but there both odors are negatively reinforced. Different odors and active port locations were attached to each contextually distinct episode. In the experimental phase, while rats were performing routine sessions in a relatively poor contextual environment in which no odors or reinforcement were delivered and all ports could provide water, we introduced on separate days two episodes, E1 and E2, containing distinct odor-place-context information.

To closely match the conditions we used in parallel to investigate human episodic memory (Saive et al., 2013, 2014), we first assessed the impact of episode repetition on the strength of 
A

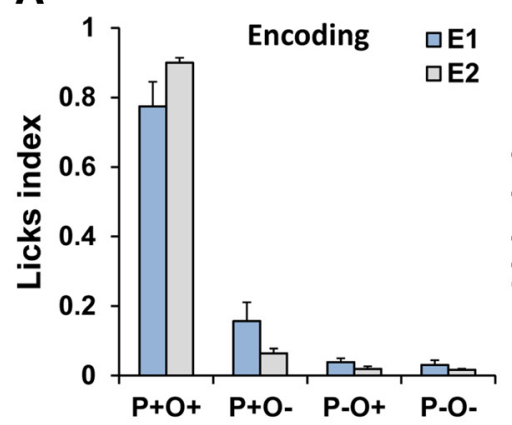

D

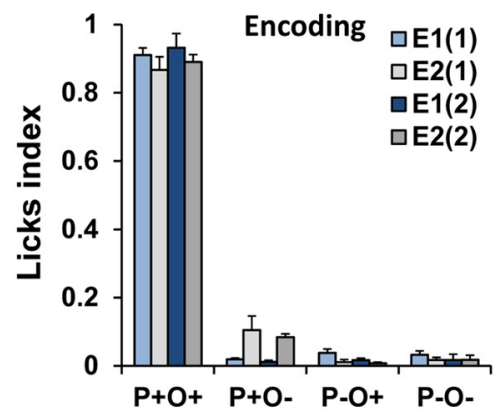

B

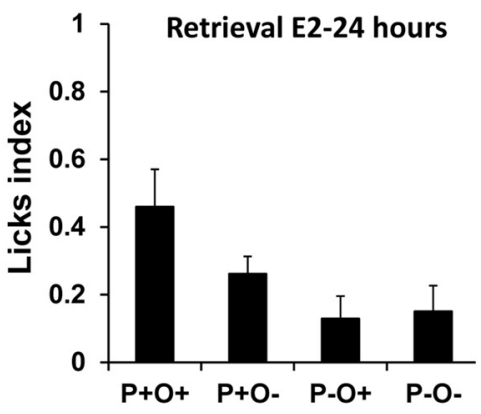

C

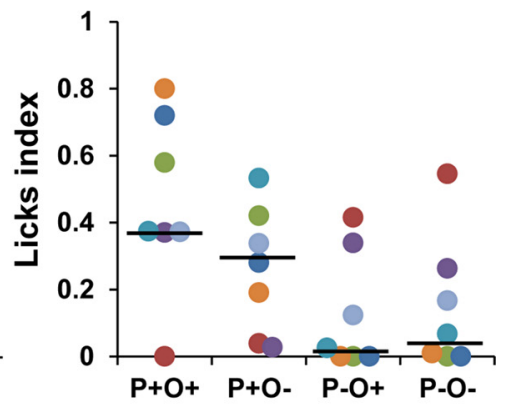

E

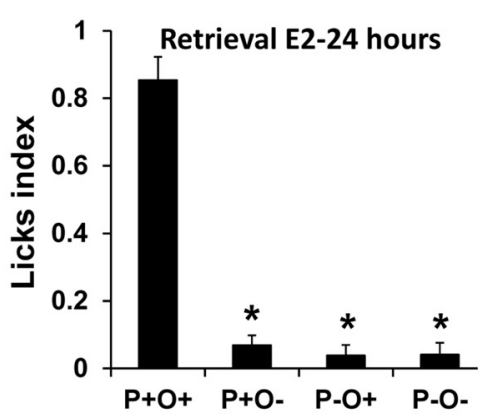

F

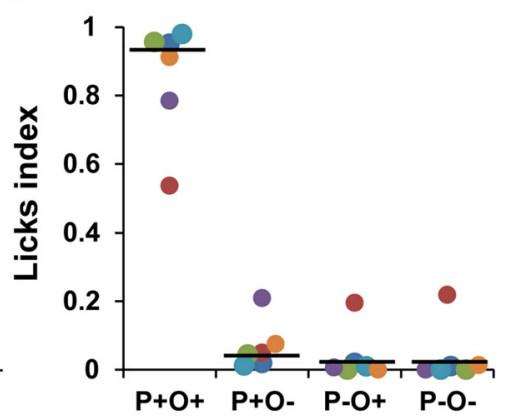

G

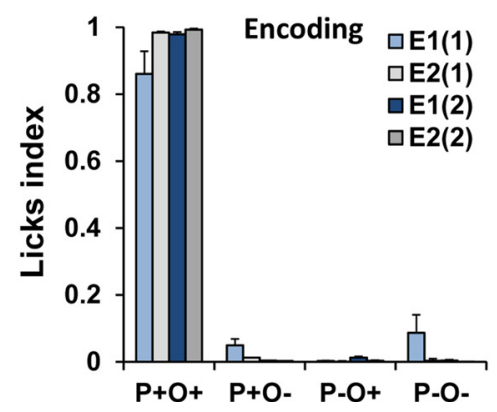

H

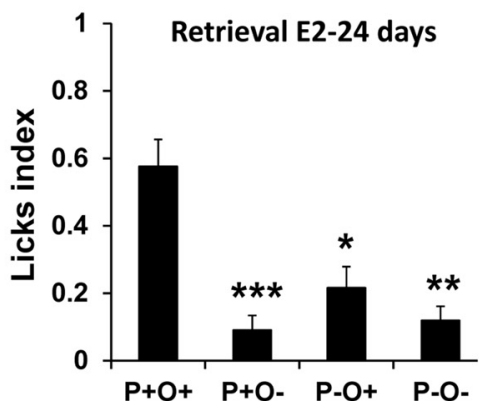

I

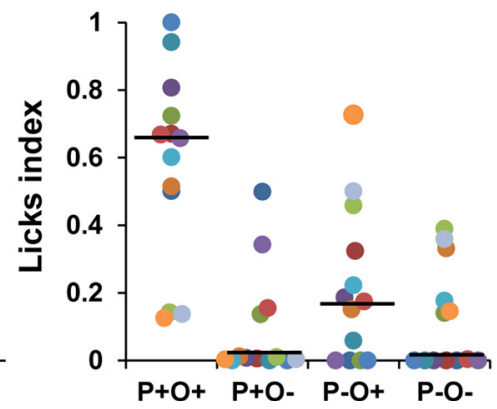

Figure 3. Episodic-like memory performance after one or two presentations of the episodes. $\boldsymbol{A}-\boldsymbol{I}, 0$ ne $(\boldsymbol{A}-\boldsymbol{C})$ and two $(\boldsymbol{D}-\boldsymbol{I})$ presentations of each episode. Licks index for each odor-placecontext association $(P+0+$ : correct odor, correct place; $P+0-$ : correct port, incorrect odor; $P-0+$ : incorrect port, correct odor; $P-0-:$ incorrect odor, incorrect port) during encoding of $E 1$ and E2 $(\boldsymbol{A}, \boldsymbol{D})$ and during $\mathrm{E} 2$ retrieval at test $24 \mathrm{~h}(\boldsymbol{B}, \boldsymbol{E})$ or 24 d later $(\boldsymbol{G}) . \boldsymbol{C}, \boldsymbol{F}, \boldsymbol{I}$, Individual licks index values during $\mathrm{E} 2$ retrieval test $(\boldsymbol{C}, n=7 ; \boldsymbol{F}, n=6 ; \boldsymbol{I}, n=13)$. Licks index values for each configuration are color coded for each individual rat. For example, the rat corresponding to the orange circle is classified as able to recall what-where information from the right context, whereas the light blue one is able to recall where information. Group data are expressed as means \pm SEM in this and subsequent figures. Median is reported as horizontal bar. ${ }^{*} p<0.05 ;{ }^{* *} p<0.01 ;{ }^{* * *} p<0.005$, Friedman test followed by Wilcoxon test.

"what-where-in which context" memory. For this, we compared performance of two independent groups of rats submitted to either one or two presentations of E1 and E2 and tested $24 \mathrm{~h}$ later for retention of E2 (Fig. 2B). As expected, during E1-E2 exposures, the two groups quickly encoded that in one specific context, in one port $(\mathrm{P}+)$ only one odor $(\mathrm{O}+)$ was associated with sugar solution delivery: they preferentially licked when they encountered the $\mathrm{P}+\mathrm{O}+$ configuration associated with sugar, whereas they avoided quinine in all other configurations $(\mathrm{P}+\mathrm{O}-, \mathrm{P}-\mathrm{O}+, \mathrm{P}-\mathrm{O}-$; Fig. $3 A ; p<0.05$ Friedman test for each E1 and E2 sessions), and behaved similarly for the two episodes (Fig. 3A; $\mathrm{P}+\mathrm{O}+$, E1 vs E2, $p=0.08$, Mann-Whitney test, $n=7)$. Similar results were obtained when rats were exposed twice to the episodes (Fig. 3D; E1(1)/E2(1)/E1(2)/E2(2) comparison $p=0.24$, Kruskall-Wallis test, $n=6$ ). During exposure to the episodes, the number of visits on each port during successive E1/E2 episodes increased progressively to $\mathrm{P}+$ and decreased to
$\mathrm{P}-$, suggesting rats progressively shaped their responses toward acquiring the place-context association (data not shown; Friedman test [E1(1), $p=0.10$; E2(1), $p=0.003$; E1(2), $p=0.016$; $\mathrm{E} 2(2), p=0.0024])$.

The critical test was then to evaluate what the rats were able to recall from their past experience. For this, a single probe test was conducted within the E2 context. However, only water was delivered (Fig. 2B, Test E2-2 ports). The licks index on each port represents what rats expect to drink and consequently reflects their episodic-like memory performance. When rats have been exposed to only one presentation of each episode, analysis of performance showed a higher licking rate in the configuration corresponding to the odor-place-context association (Fig. 3B). Although the global group performance on $\mathrm{P}+\mathrm{O}+$ against the other configurations only showed a trend toward statistical significance ( $p=0.074$, Friedman test), analyses of individual performance revealed distinct individual behavioral profiles (Fig. 
$3 C)$ with nearly a third (29\%) of the rats presenting "whatwhere" behavior selective of context E2, the correct integrated episodic-like memory (Table 1). Other individuals were categorized as "where" (43\%), "what" (14\%), or indeterminate behavior (14\%). Further, when rats were exposed twice to each episode, they were clearly all able to remember the entire combination of episodic information (Fig. 3E; $p=0.0052$; Friedman test; $\mathrm{P}+\mathrm{O}+$ vs the other combinations, $p=0.027$, Wilcoxon test), $100 \%$ of them exhibiting the correct odor-place-context behavioral profile (Fig. 3F; Table 1). Because rats during encoding were similarly exposed to $\mathrm{O}+$ and $\mathrm{O}-$ at $\mathrm{P}+$ (data not shown; $p=0.46$, Wilcoxon test), their memory ability in recollecting the episode cannot be explained by a biased experience for the $\mathrm{P}+\mathrm{O}+$ configuration. Statistical comparison of the two experimental groups [one exposure (Fig. 3B) vs two exposures (Fig. 3E)] showed a significant group difference in recall performance $(p<0.0001$, $\chi^{2}$ test; $p=0.0152$, Mann-Whitney test on $\mathrm{P}+\mathrm{O}+$ ). By contrast, performance of "what-where" rats in the two groups (comparison of licks index on $\mathrm{P}+\mathrm{O}+$ of the two groups) was not statistically significant ( $p=0.32$, Mann-Whitney test), suggesting no qualitative difference in performance in good performers whether they had been exposed once or twice to the two episodes.

These findings indicate that when rats were confronted to the two brief and distinct episodes only once, nearly a third remembered the combined "what-where-in-which-context" information, while two presentations of the two episodes allowed all rats to accurately recollect an integrated memory of the entire episodic information. Notably, the present odor-associated episodic memory paradigm reveals in rats an inherent variability of recollection profiles, which is similar to what we reported in humans (Saive et al., 2013, 2014), offering the possibility to investigate the neural substrate of information each animal was able to recollect.

Next we examined recollection performance at the much longer exposure-to-test interval of $24 \mathrm{~d}$ in an independent group of rats after they had been exposed twice to the two different episodes E1 and E2 (Fig. 2). Again, during E1-E2 exposures, rats preferentially licked when they encountered the $\mathrm{P}+\mathrm{O}+$ configuration associated with sugar, whereas they avoided quinine in all other configurations $(\mathrm{P}+\mathrm{O}-, \mathrm{P}-\mathrm{O}+, \mathrm{P}-\mathrm{O}-$; Fig. $3 G$; $p<$ 0.001 , Friedman test for each E1 and E2 sessions), thus replicating the above results. Twenty-four days after training, 8 of 13 rats $(62 \%)$ were still able to remember the entire combination of episodic information (Fig. $3 H ; p=0.001$, Friedman test; $\mathrm{P}+\mathrm{O}+$ vs the other combinations, $p<0.02$, Wilcoxon test), exhibiting the correct odor-place-context behavioral profile (Fig. 3I). Thus, even with relatively limited exposure to two complex episodes, rats are able to form a robust and long-lasting episodic-like memory that they can recall $24 \mathrm{~d}$ after exposure to the episodes.

\section{Resolution of episodic-like memory recollection in a challenged situation of recall}

In many circumstances of daily life, remembering episodes occurs in contexts that are not fully identical to the encoding situation. To test this property and evaluate the strength and resolution of episodic-like memory recollection, we challenged memory retrieval by placing animals in a high-interference situation during recall. After having experienced two presentations of each episode, animals were now tested $24 \mathrm{~h}$ later in a four-port E2 test (Fig. 2B, Test E2-4 ports). In this case, rats had to recollect the odor-place association of E2 (IC), while ignoring the odorplace information previously associated with E1 (distracting ports; OC). Analysis of individual recollection profiles showed
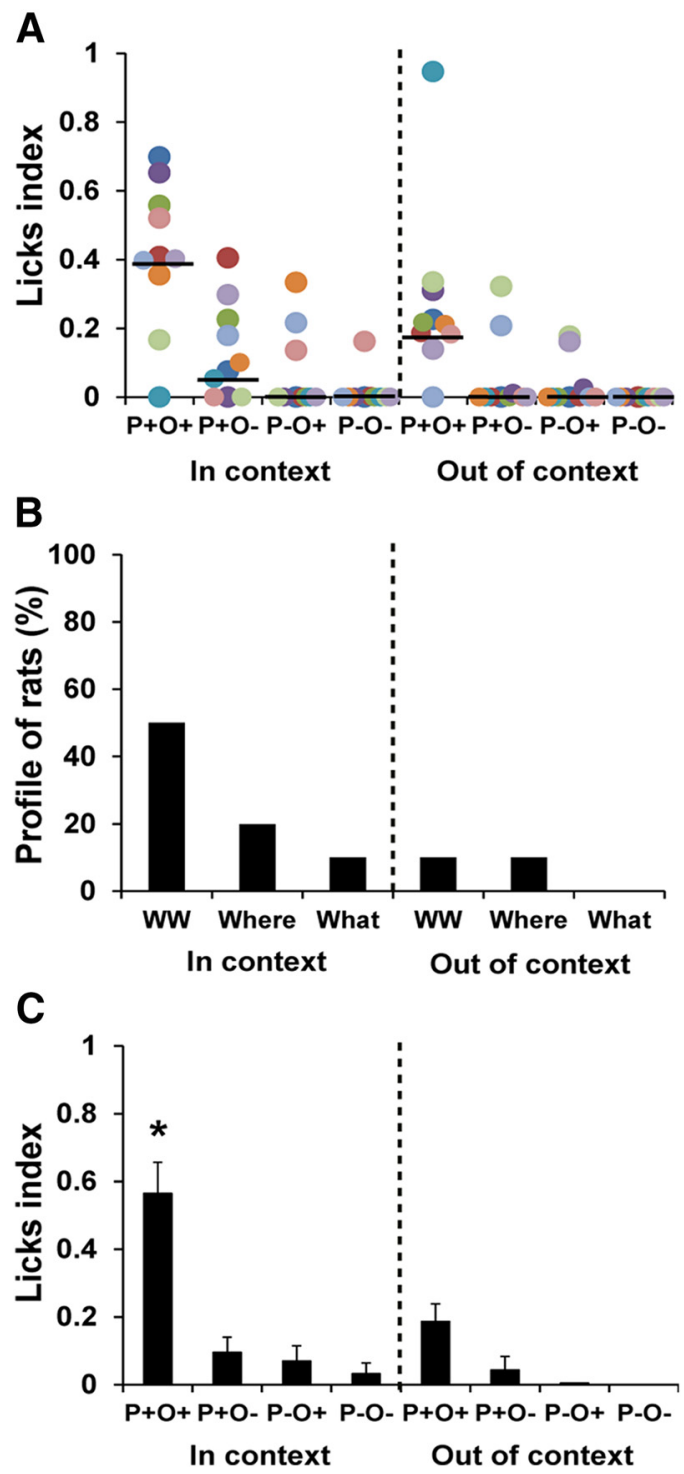

Figure 4. Episodic-like memory performance in E2 context during the challenging four-port test. $\boldsymbol{A}$, Individual licks index for each configuration IC or $0 C(n=10)$. $\boldsymbol{B}$, Distribution of individual behavioral profiles during recollection of the episodic association. Histograms represent What-Where (WW), Where, What profiles IC or OCE2 $(n=10)$. C, Licks index of rats expressing a What-Where-IC profile $(n=5$ of 10). The results of the $P+0+/ I C$ configuration was significantly different from all the other configurations, ${ }^{*} p<0.05$, Friedman test followed by Wilcoxon test.

that memory performance, despite a certain degree of variability, remained reliable and robust, suggesting that rats were able to disambiguate the information on display during recall (Fig. 4A; Table $2, n=10)$. In fact, $50 \%$ of the rats exhibited a whatwhere-IC profile (Fig. 4B), displaying a significantly higher licks index in $\mathrm{P}+\mathrm{O}+$ configuration of the tested context (Fig. 4C; $\mathrm{P}+\mathrm{O}+/ \mathrm{IC}$ vs the other combinations, $p=0.028$, Wilcoxon test, $n=5$ ). Moreover, $80 \%$ of the rats were able to recollect the association between the ports and the contextual cues of the tested episode (Fig. 4B, In context). Among those, the majority (62.5\%; what-where-IC) retrieved the whole episode, while a few were only able to recollect the place $(25 \%$; where-IC) or the odor (12.5\%; what-IC). Thus, a high proportion of animals formed an integrated episodic-like memory that was sufficiently accurate, robust, and flexible to face a highly interfering retrieval situation, 
a key feature of human memory to dissociate different personal past experiences.

Episodic-like memory recall requires an intact hippocampus The hippocampus is critical for episodic memory in humans (Tulving and Markowitsch, 1998; Eichenbaum et al., 2007) and is also required to encode and recall episodic-like memory in animals (Ergorul and Eichenbaum, 2004; Fortin et al., 2004; Eichenbaum and Fortin, 2005). Thus, we expected that inactivation of the hippocampus during recall would impair recollection of an integrated account of the episodic information in our paradigm. We temporarily inactivated the dorsal hippocampus by infusion of muscimol 40 min before E2 retrieval. Control animals (operated noninjected and aCSF injected) exhibited similar recall performance (data not shown; $p=0.73$ Mann-Whitney test) and were therefore pooled. Animals later assigned to the control or muscimol groups similarly exhibited a preference for $\mathrm{P}+\mathrm{O}+$ versus the other configurations during the encoding of episode E2 (Fig. $5 A$; control, CONT, $p=0.0001, n=10$; muscimol, MU, $p=$ $0.002, n=7$, Friedman test). However, during retrieval, muscimol rats, in contrast to controls, preferentially licked from $\mathrm{P}+$, but regardless of the odor delivered following a nose poke (Fig. $5 A ; \mathrm{P}+\mathrm{O}+$ vs $\mathrm{P}+\mathrm{O}-$, control, $p=0.009$; muscimol, $p=0.091$, Wilcoxon test; $\mathrm{P}+\mathrm{O}+$, control vs muscimol, $p=0.025$, MannWhitney test). Thus, while control rats exhibited a What-Where profile corresponding to the right context, muscimol-injected rats were strongly impaired in remembering the integrated odorplace-context association. This was confirmed by analyzing individual performance, which showed no recall of the episodic association in muscimol-injected rats (Fig. 5C) compared with the expected behavioral profile of episodic memory recollection in controls (Fig. 5B). Of note, both control and muscimol rats similarly made more frequent visits to the reinforced port $\mathrm{P}+$ than to the nonreinforced port $\mathrm{P}-$ (percentage of visits $\mathrm{P}+$ : control, $87.5 \pm 3.3$; muscimol, $86.9 \pm 4.8$; percentage of visits $\mathrm{P}-$ : control, $12.5 \pm 3.34$; muscimol, $13.1 \pm 4.8 ; \mathrm{P}+$ vs $\mathrm{P}-$ : control, $p=0.005$; muscimol, $p=0.018$, Wilcoxon test; control vs muscimol on $\mathrm{P}+: p=0.99$, Mann-Whitney test), demonstrating that the "where" component of the episodic association is robust, even under hippocampal blockade, a logical consequence of the paradigm as port location is the first information captured before a decision is made to respond or not to the odor. Overall, these results validate the premise that complete recollection of the integrated episodic information in our paradigm strongly depends on hippocampal function.

\section{Recruitment of selective brain areas during recollection of episodic events}

To identify brain regions involved in processing episodic-like memory, we used imaging of the expression of the activitydependent immediate early genes (IEGs), c-fos and zif268, two markers widely used to map neural ensembles activated by experience and known to be required for synaptic plasticity and longterm memory (Jones et al., 2001; Fleischmann et al., 2003; Veyrac et al., 2013, 2014). IEG expression was investigated $90 \mathrm{~min}$ after retrieval in rats exposed to the episodes (episodic rats, $n=6$ ) and compared with controls exposed to routine sessions only (routine rats, $n=6$; Fig. 6). We found significant increases in the number of c-Fos-immunoreactive neurons in dorsal (Figs. $6 B, D$ ) and ventral (Fig. $6 C$ ) areas of the hippocampus associated with a concomitant increase in orbitofrontal cortex (OFC; Fig. 6E), PFC, and aCC (Fig. 6F). Zif268 expression was also increased in the same areas (Fig. $6 H, I, K, L$ ), except in OFC, in which Zif268
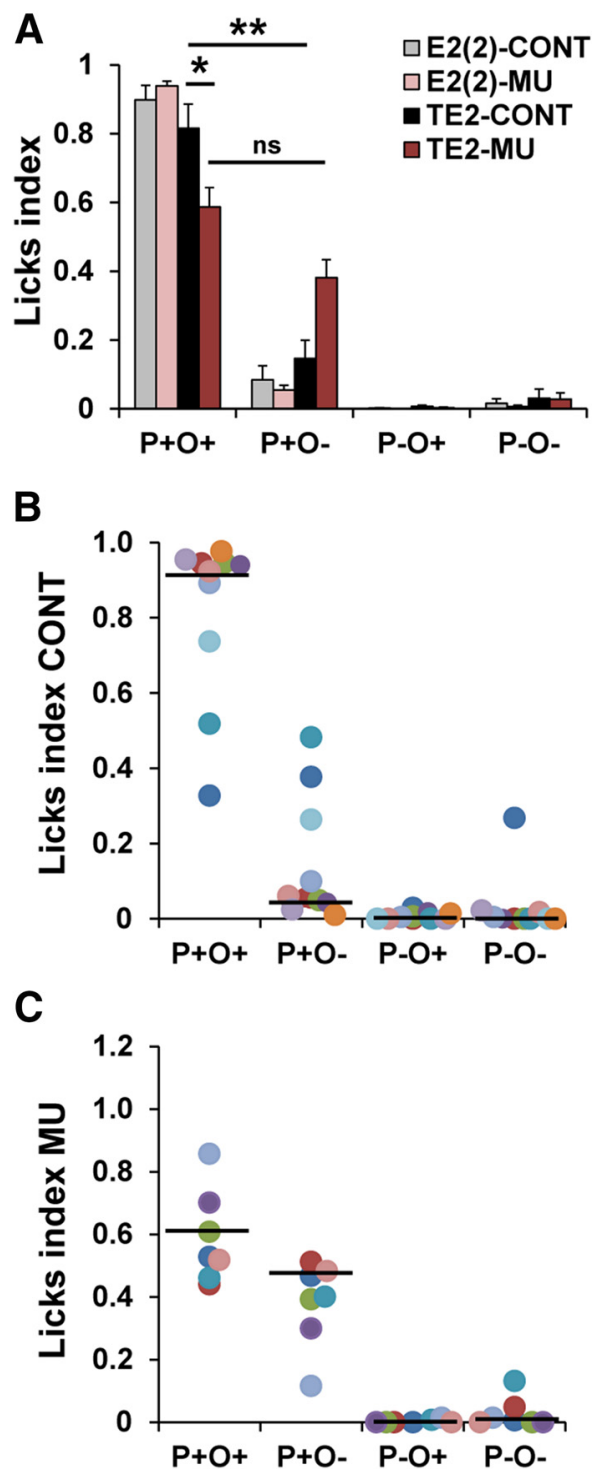

Figure 5. Episodic-like memory performance during transient pharmacological inactivation of the dorsal hippocampus. $\boldsymbol{A}$, Licks index for each odor-place-context configuration during encoding of the second session of E2 [E2(2)] and during E2 retrieval test (TE2) in control rats (CONT; $n=10)$ and rats injected with muscimol (MU; $n=7)$ before the retrieval test. $B, C$, Individual licks index values of CONT $(\boldsymbol{B})$ and MU $(\boldsymbol{C})$ rats during E2 retrieval test. Medians for each configuration are reported on the graphs. ${ }^{*} p<0.05,{ }^{* *} p<0.01$. ns, Nonsignificant. Friedman test followed by Wilcoxon test or Mann-Whitney test for comparisons of independent groups.

expression was similar for episodic and routine rats (Fig. $6 K$ ). Notably, the absence of observable activation in primary olfactory and lateral entorhinal cortices (piriform cortex: Fos, routine rats, $0.53 * 10^{-3}$ cells $/ \mu \mathrm{m}^{2}$; episodic rats, $0.57 * 10^{-3}$; Zif268, routine rats, $1.25^{\star} 10^{-3}$; episodic rats, $1.17^{*} 10^{-3}$; lateral entorhinal cortex: Fos, routine rats, $0.25 * 10^{-3}$ cells $/ \mu \mathrm{m}^{2}$; episodic rats, $0.23^{\star} 10^{-3}$; Zif268, routine rats, $0.26^{\star} 10^{-3}$; episodic rats, $0.28^{\star} 10^{-3}$ ), suggests that odor processing per se is not a determinant process engaged at recall of the episode, and implies that the OFC participates in retrieval of the integrated memory rather than simply in odor processing. We observed no induction of c-Fos or Zif268 in the perirhinal cortex (data not shown), suggesting that performance in this task is not primarily based on familiarity detection (Brown and Aggleton, 2001). Thus, although brain regions in which recollection-related expression of 
A
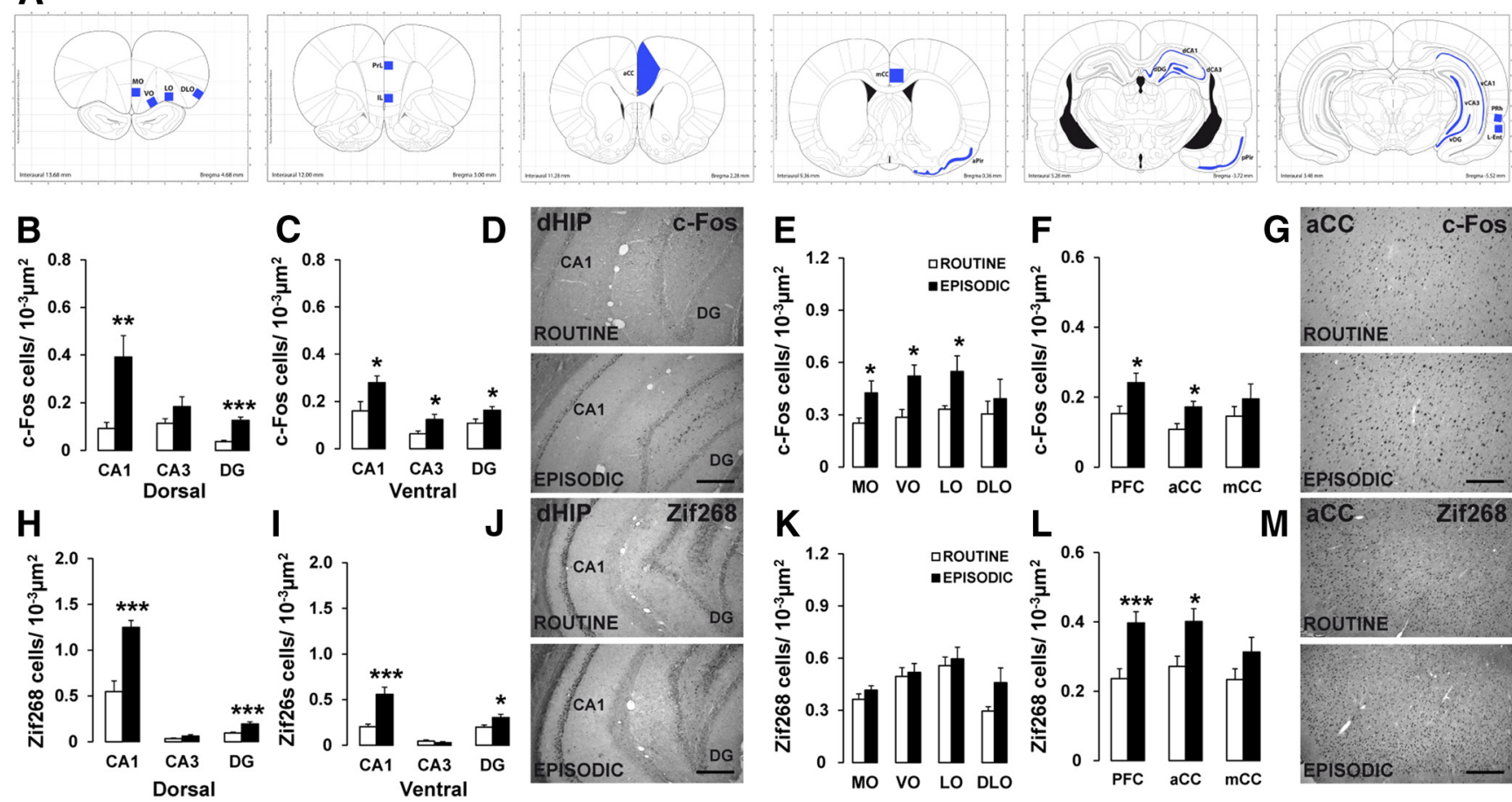

Figure 6. Recruitment of selective brain areas during recollection of the episodic association. $A$, Schematic drawings of rat brain coronal sections showing the regions of interest (filled areas) selected for counting of IEG-positive nuclei. Distance from bregma is indicated for each coronal section. $\boldsymbol{B}-\boldsymbol{M}, \boldsymbol{C}-\mathrm{Fos}(\boldsymbol{B}-\boldsymbol{G})$ and Zif268 $(\boldsymbol{H}-\boldsymbol{M})$ cell densities in dorsal $(\boldsymbol{B}, \boldsymbol{H})$ and ventral $(\boldsymbol{C}, \boldsymbol{I})$ hippocampus; medial (MO), ventral (V0), lateral ( $(0)$, and dorsolateral (DL0) parts of orbitofrontal (OFC) cortex $(\boldsymbol{E}, \boldsymbol{K})$; and PFC, aCC, $\mathrm{mCC}(\boldsymbol{F}, \boldsymbol{L})$. Histograms represent c-Fos and Zif268 cell counts in control (white bars, ROUTINE; $n=6$ ) and episode-experienced rats (black bars, EPISODIC; $n=6) .{ }^{*} p<0.05,{ }^{* *} p<0.01,{ }^{* * *} p<0.005$, Student's $t$ test. D, G, J, M, Photomicrographs showing increased c-Fos and Zif268 expression in episodic rats compared with control rats in dorsal hippocampus (D,J) and in aCC $(\boldsymbol{G}, \boldsymbol{M})$. Scale bars: $\mathbf{G}, 250 \mu \mathrm{m} ; \boldsymbol{D}, \boldsymbol{J}, \boldsymbol{M}, 500 \mu \mathrm{m}$.

A

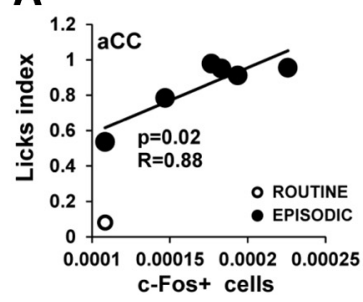

B

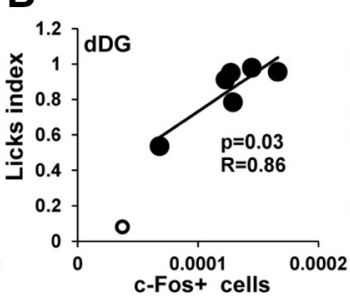

C

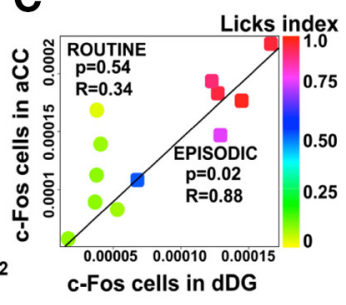

D

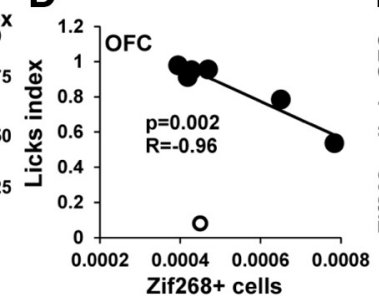

E

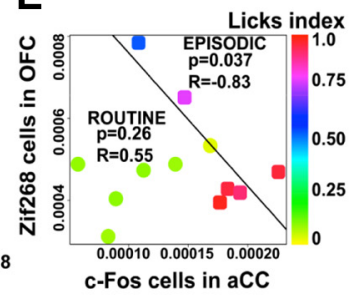

Figure 7. Episodic-like memory performance correlates with the recruitment of prefrontal and hippocampal areas. $A, B, D$, Pearson's $r$ correlation between licks index and c-Fos expression in aCC $(\boldsymbol{A})$ and dDG $(\boldsymbol{B})$ and between licks index and Zif268 expression in OFC (D). C, Pearson's cross-correlation between c-Fos expression in aCC and dDG and licks index. $\boldsymbol{E}$, Pearson's cross-correlation between c-Fos expression in aCC and Zif268 expression in OFC and licks index. Control (ROUTINE) rats are represented with circles and episodic rats (EPISODIC) by squares. Right color scales in C and $\boldsymbol{E}$ indicate licks index level during the E2 recall test for each individual data point. Black line, Regression line for episodic rats.

IEGs were not detected could nevertheless participate in memory processing and recall, these findings reveal a core-distributed neural network recruited during recall of long-term episodic-like memory that involves a dialog between hippocampal and PFC association areas. This neural circuit is highly similar to that identified in human brain imaging studies as a potential network underlying episodic memory recollection (Diana et al., 2007; Daselaar et al., 2008).

\section{Correlation of brain activation and episodic-like memory performance}

In memory tasks, the behavioral measure used as an index of performance is critical. The present paradigm allows precise quantification of licking behavior in each experimental configuration, a direct operational measure of the ability to recollect the what-where-in-which-context episodic association. The licks index also allows the categorization of individual recollection pro- files, an ideal situation for investigating correlations between recruitment of selective brain regions (Fig. 6) and accuracy of retrieval performance (Fig. 3F). Among brain regions recruited during memory recollection, significant correlations between c-Fos expression and the memory performance index were selectively obtained in aCC and dDG of the hippocampus (Fig. $7 A, B$ ); that is, higher activation of c-Fos in both aCC $(n=6, r=0.88$, $F_{(1,4)}=16.10, p=0.02$, Pearson's correlation) and dDG $(n=6$, $\left.r=0.86, F_{(1,4)}=14.22, p=0.03\right)$ was commensurate with the most faithful licking performance on $\mathrm{P}+\mathrm{O}+$ during retrieval. A further cross-correlation analysis revealed that levels of activation of these two areas were closely related to each other and increased monotonically with performance levels for the correct episodic association $\left(n=6, r=0.88, F_{(1,4)}=13.44, p=0.02\right.$; Fig. $7 C$, squares). No such correlation was found in control rats $(n=$ $6, r=0.34, F_{(1,4)}=0.52 ; p=0.54$; Fig. $7 C$, circles). To control for the specificity of activation of these structures in relation to 
episodic-like memory performance, we analyzed in the same way data from routine rats and found no significant correlation (data not shown; $n=6$; aCC: $r=-0.71, F_{(1,4)}=3.93, p=0.11$; dDG: $\left.r=-0.15, F_{(1,4)}=0.087, p=0.76\right)$. Of note, there was also no significant correlation between c-Fos expression and the total number of licks made by episodic rats during recall, regardless of the configurations, thus excluding the possibility of a bias on correlations due to drinking behavior (data not shown; $n=6$; aCC: $r=-0.54, F_{(1,4)}=1.81, p=0.27$; dDG: $r=-0.16, F_{(1,4)}=$ $0.095, p=0.76)$.

Our analyses additionally revealed a negative correlation between performance of episodic rats and Zif268 expression levels in subareas of the OFC (Fig. $7 D ; n=6, r=-0.96, F_{(1,4)}=48.88$, $p=0.002$ ), while no such relationship was observed in association with drinking behavior or in routine rats (data not shown; $n=6, r=0.40, F_{(1,4)}=0.78, p=0.43 ; r=-0.39, F_{(1,4)}=0.74$, $p=0.43)$. Although there was no overall significant increase in Zif268 expression in OFC for the episodic group after recall (Fig. $6 \mathrm{~K})$, this nevertheless indicates more OFC Zif268 expression levels in rats that perform less well. This is further illustrated by a negative correlation between OFC Zif268 expression and c-Fos expression in aCC in relation to memory performance (Fig. 7E, EPISODIC, squares: $n=6, r=-0.83, F_{(1,4)}=9.32, p=0.037$; ROUTINE, circles: $n=6, r=0.55, F_{(1,4)}=1.69, p=0.26$ ). Collectively, these findings strongly support the idea that the strength of hippocampal-PFC engagement during recall reflects the accuracy of episodic recollection performance during recall.

\section{Discussion}

A primary challenge in the search for the mechanisms underlying complex forms of human memory, such as episodic memory, is to develop adequate animal models amenable to neurobiological investigation. Here, we present an entirely novel paradigm developed in parallel with a task used in humans (Saive et al., 2013, 2014), in which subjects freely explored brief daily episodes composed of different odors at specific locations within distinct visual contexts, to evaluate encoding and recollection of what-wherein-which-context/occasion episodic-like memory in laboratory rats. With this paradigm, we demonstrate that after limited exposure to two different episodes introduced during their daily life, rats, like humans, are able to recollect with individual abilities a unified and robust long-term memory of what happened where on a unique and specific contextual occasion. We confirm that memory recollection in this task requires an intact hippocampus and report that recollection is associated with the recruitment of a specific, distributed hippocampal-PFC network that correlates with the accuracy of memory performance.

The task designed for human participants (Saive et al., 2013, 2014) and the present study in rats present many similarities: each episode was characterized by olfactory stimuli presented in specific spatial locations in a rich environmental context. Episodes were presented on distinct days and during encoding both humans and rats were allowed to freely explore the environment. There were also certain differences, in particular during retrieval: rats, placed in the context of a given episode, must first grasp and process this multidimensional information, then select a place to get odorant information, and then, according to the olfactory stimulus delivered at this place (two different possibilities), to decide to drink or not. This decision indicated whether they remembered the what-where association specific to this context. In the task designed for humans, on each trial an olfactory stimulus was delivered out of the context and participants were asked whether they already had perceived it, and, if yes, then placed it correctly in the right context. These two retrieval situations differed in the first cue used to trigger episodic recollection: the context in rats and the odor in humans. However, when episodic recollection performance was compared in both tasks in the same condition (one exposure of each episode), the proportion of episodic recollection was $\sim 30 \%$. Thus, at least in this task, rats and humans shared similar profiles of episodic memory formation and recollection.

Previous studies with rodents addressing the feasibility to assess episodic-like memory using odor cues mainly focused on the ability to remember odor-place sequences (Ergorul and Eichenbaum, 2004). Successful performance provided evidence that rats can combine what/where cues and remember in which order events have been experienced, at least as tested at very short learning-to-retention delays (7 s); however, this paradigm requires rats to learn first an explicit rule by extensive training, which human episodic memory does not require (Tulving, 2002). Here, the way we designed our paradigm allowed minimizing such training effects to more closely match the characteristics of human episodic memory and to engender long-term memory. In that sense, our protocol resembles more certain objectrecognition tasks (Eacott et al., 2005), with the determining distinctiveness that we placed emphasis on the ability to separate different components of episodic information the animal can use, an impracticable option in object-recognition tasks. Moreover, in odor-place sequence tasks, sampling odors requires first to go to a given place, and "what" and "where" elements are thus tightly linked. To avoid this, we designed the task so that animals were exposed to the same odor in different places, and to different odors in each place, an important aspect to disambiguate the nature of the recollected information.

Moreover, the present task based on searching for a rewarding drinking solution offers the means to measure recollection accurately and discern the animal's expectation by directly evaluating what did remain in memory of a given occasionally encountered contextual situation. Further, in contrast to all other episodic-like memory tasks in rodents, this paradigm allows evaluating both long-term and flexible memory in a challenged situation of recall, two main properties of human episodic memory.

One potential compromising factor was the particularly high risk of poor encoding or forgetting, because of the brief exposures and degree of overlap between the two episodes despite the differences in certain contextual information, odors, and locations of the rewarded ports. However, even with a unique exposure of each episode, most rats were able to recollect which place was associated with sugar and nearly a third were able to recollect the combined what-where-in-which-context information. Notably, this inherent degree of variability in the ability of rats to encode or recall the combined settings is strikingly similar to what has been reported in humans, since in an analogous odor-place-context episodic memory task in human subjects the score for a full episodic recollection is $30 \%$ on average (Saive et al., 2013, 2014). Crucially, only one repetition of the episodes was sufficient to increase the number of rats that can accurately recollect all relevant information related to the episode $24 \mathrm{~h}$ after. Moreover, even $24 \mathrm{~d}$ after exposures to the episodes, accurate episodic recollection was still observed in $62 \%$ of the animals. This establishes that rats are able to form a robust and long-lasting episodic-like memory. Moreover, when tested in a more challenging situation (four-port test, with distracting odor-port combinations that relate to distinct encoded episodes), $80 \%$ of the animals showed correct recollection of the place and context association and 63\% of them were able to accurately retrieve all the information con- 
tent of the episode. These results confirm that a large proportion of rats had kept a long-term memory of all the elements of the episodes and that, when facing an interfering situation in which elements common to different past episodes are present, they can recall a specific episode on the basis of the context, thus showing their capacity to recall an episodic memory flexibly, a key attribute of episodic memory (Clayton et al., 2003).

It is widely held that the hippocampus is required for recall of episodic memory (Tulving and Markowitsch, 1998; Ergorul and Eichenbaum, 2004; Fortin et al., 2004; Eichenbaum and Fortin, 2005; Eichenbaum et al., 2007). To validate our task, we transiently inactivated the hippocampus during recall and found this indeed prevented recollection of the integrated what-where-inwhich-context information. We then explored what brain circuits are engaged when the memory is reactivated during recall. For this, we mapped IEG activation in numerous brain areas after recall and found preferential, coordinated activation of hippocampal and PFC areas that correlated with the accuracy of episodic-like memory recollection across subjects.

Animal studies have to our knowledge never evaluated neural network recruitment in episodic-like memory, and in investigations of other forms of memories, distinct patterns of expression of different IEGs were often observed (Tse et al., 2011; Barbosa et al., 2013), probably because these IEGs have different functions (Davis et al., 2003). c-Fos is generally believed to reflect eventinduced neuronal activation, while the transcription factor Zif268 more likely reflects synaptic activation and plasticity mechanisms involved in long-term memory (Jones et al., 2001; Veyrac et al., 2014). Although the precise function of each brain area engaged remains to be elucidated, our imaging data provide evidence for the involvement of a distributed hippocampalmedial PFC network in long-term episodic-like memory and for a role of the hippocampus as a crucial recollection organizing module (Eichenbaum et al., 2007), as previously reported for other types of olfactory associative memories (Lesburguères et al., 2011; Tse et al., 2011). A particular key role of the DG is highlighted, possibly as a key structure involved in pattern-separation function (McHugh et al., 2007; Clelland et al., 2009) related to episodic-like memory (Yassa and Reagh, 2013). Importantly, these brain structures are to a large extent similar to those found by functional MRI to be activated in humans following recall of episodic memory (Diana et al., 2007; Daselaar et al., 2008). Reinforcing the idea that recruitment of this network serves recollection is the finding that specific activation of subareas of this hippocampal-PFC network is predictive of faithful recall of the integrated episodic-like memory.

In conclusion, using this novel paradigm, the present findings provide evidence that rats can form an episodic-like memory with features that satisfy several important criteria attached to human episodic memory in terms of informational content, limited occasional encounter of episodes, encoding and long-term recollection of an integrated what-where-in-which-context information, categorization of individual profiles of recollection, and the capacity for flexible use of this memory. Our results also demonstrate that recall of episodic-like memory recruits a distributed network of hippocampal-PFC structures that correlates with episode recollection performance. This model in conjunction with other neurobiological techniques offers the possibility to investigate genetic, molecular, and neurophysiological mechanisms, and network dynamics that underlie both episodic memory encoding and faithful recollection of specific past events and to further our understanding of the mechanisms that go awry in models of human conditions associated with episodic memory disorders.

\section{References}

Barbosa FF, Santos JR, Meurer YS, Macêdo PT, Ferreira LM, Pontes IM, Ribeiro AM, Silva RH (2013) Differential cortical c-Fos and Zif-268 expression after object and spatial memory processing in a standard or episodic-like object recognition task. Front Behav Neurosci 7:112. CrossRef Medline

Brown MW, Aggleton JP (2001) Recognition memory: what are the roles of the perirhinal cortex and hippocampus? Nat Rev Neurosci 2:51-61. CrossRef Medline

Clayton NS, Dickinson A (1998) Episodic-like memory during cache recovery by scrub jays. Nature 395:272-274. CrossRef Medline

Clayton NS, Bussey TJ, Dickinson A (2003) Can animals recall the past and plan for the future? Nat Rev Neurosci 4:685-691. CrossRef Medline

Clelland CD, Choi M, Romberg C, Clemenson GD Jr, Fragniere A, Tyers P, Jessberger S, Saksida LM, Barker RA, Gage FH, Bussey TJ (2009) A functional role for adult hippocampal neurogenesis in spatial pattern separation. Science 325:210-213. CrossRef Medline

Daselaar SM, Rice HJ, Greenberg DL, Cabeza R, LaBar KS, Rubin DC (2008) The spatiotemporal dynamics of autobiographical memory: neural correlates of recall, emotional intensity, and reliving. Cereb Cortex 18:217229. CrossRef Medline

Davis S, Bozon B, Laroche S (2003) How necessary is the activation of the immediate early gene zif268 in synaptic plasticity and learning? Behav Brain Res 142:17-30. CrossRef Medline

Diana RA, Yonelinas AP, Ranganath C (2007) Imaging recollection and familiarity in the medial temporal lobe: a three-component model. Trends Cogn Sci 11:379-386. CrossRef Medline

Eacott MJ, Easton A (2010) Episodic memory in animals: remembering which occasion. Neuropsychologia 48:2273-2280. CrossRef Medline

Eacott MJ, Easton A, Zinkivskay A (2005) Recollection in an episodic-like memory task in the rat. Learn Mem 12:221-223. CrossRef Medline

Easton A, Eacott MJ (2008) A new working definition of episodic memory: replacing "when" with "which" In: Handbook of episodic memory (Dere E, Easton A, Nadel L, Huston JP, eds), pp 185-196. Amsterdam: Elsevier.

Easton A, Eacott MJ (2010) Recollection of episodic memory within the medial temporal lobe: behavioural dissociations from other types of memory. Behav Brain Res 215:310-317. CrossRef Medline

Eichenbaum H, Fortin NJ (2005) Bridging the gap between brain and behavior: cognitive and neural mechanisms of episodic memory. J Exp Anal Behav 84:619-629. CrossRef Medline

Eichenbaum H, Yonelinas AP, Ranganath C (2007) The medial temporal lobe and recognition memory. Annu Rev Neurosci 30:123-152. CrossRef Medline

Ergorul C, Eichenbaum H (2004) The hippocampus and memory for "what," "where," and "when." Learn Mem 11:397-405. CrossRef Medline

Fellini L, Morellini F (2013) Mice create what-where-when hippocampusdependent memories of unique experiences. J Neurosci 33:1038-1043. CrossRef Medline

Fleischmann A, Hvalby O, Jensen V, Strekalova T, Zacher C, Layer LE, Kvello A, Reschke M, Spanagel R, Sprengel R, Wagner EF, Gass P (2003) Impaired long-term memory and NR2A-type NMDA receptor-dependent synaptic plasticity in mice lacking c-Fos in the CNS. J Neurosci 23:91169122. Medline

Fortin NJ, Wright SP, Eichenbaum H (2004) Recollection-like memory retrieval in rats is dependent on the hippocampus. Nature 431:188-191. CrossRef Medline

Jones MW, Errington ML, French PJ, Fine A, Bliss TV, Garel S, Charnay P, Bozon B, Laroche S, Davis S (2001) A requirement for the immediate early gene Zif268 in the expression of late LTP and long-term memories. Nat Neurosci 4:289-296. CrossRef Medline

Lesburguères E, Gobbo OL, Alaux-Cantin S, Hambucken A, Trifilieff P, Bontempi B (2011) Early tagging of cortical networks is required for the formation of enduring associative memory. Science 331:924-928. CrossRef Medline

McHugh TJ, Jones MW, Quinn JJ, Balthasar N, Coppari R, Elmquist JK, Lowell BB, Fanselow MS, Wilson MA, Tonegawa S (2007) Dentate gyrus NMDA receptors mediate rapid pattern separation in the hippocampal network. Science 317:94-99. CrossRef Medline

Saive AL, Ravel N, Thévenet M, Royet JP, Plailly J (2013) A novel experi- 
mental approach to episodic memory in humans based on the privileged access of odors to memories. J Neurosci Methods 213:22-31. CrossRef Medline

Saive AL, Royet JP, Ravel N, Thévenet M, Garcia S, Plailly J (2014) A unique memory process modulated by emotion underpins successful odor recognition and episodic retrieval in humans. Front Behav Neurosci 8:203. CrossRef Medline

Suddendorf T, Corballis MC (2007) The evolution of foresight: what is mental time travel, and is it unique to humans? Behav Brain Sci 30:299313; discussion 313-351. Medline

Templer VL, Hampton RR (2013) Episodic memory in nonhuman animals. Curr Biol 23:R801-R806. CrossRef Medline

Tse D, Takeuchi T, Kakeyama M, Kajii Y, Okuno H, Tohyama C, Bito H, Morris RG (2011) Schema-dependent gene activation and memory encoding in neocortex. Science 333:891-895. CrossRef Medline

Tulving E (1972) Episodic and semantic memory. In: Organization of memory (Tulving E, Donaldson W, eds), pp 383-403. New York: Academic.
Tulving E (2002) Episodic memory: from mind to brain. Annu Rev Psychol 53:1-25. CrossRef Medline

Tulving E, Markowitsch HJ (1998) Episodic and declarative memory: role of the hippocampus. Hippocampus 8:198-204. CrossRef Medline

Veyrac A, Gros A, Bruel-Jungerman E, Rochefort C, Kleine Borgmann FB, Jessberger S, Laroche S (2013) Zif268/egrl gene controls the selection, maturation and functional integration of adult hippocampal newborn neurons by learning. Proc Natl Acad Sci U S A 110:7062-7067. CrossRef Medline

Veyrac A, Besnard A, Caboche J, Davis S, Laroche S (2014) The transcription factor zif268/egrl, brain plasticity, and memory. Prog Mol Biol Transl Sci 122:89-129. CrossRef Medline

Yassa MA, Reagh ZM (2013) Competitive trace theory: a role for the hippocampus in contextual interference during retrieval. Front Behav Neurosci 7:107. CrossRef Medline

Zhou W, Crystal JD (2011) Validation of a rodent model of episodic memory. Anim Cogn 14:325-340. CrossRef Medline 